\title{
Viral PCR on cerebrospinal fluid samples without pleocytosis: clinically useful for definitive diagnosis in infants (rather than newborns), but leaving a dilemma for follow-up care
}

Authors: Jonathan P Sturgeon"1; Beatrice Zanetti1; Dwight Lindo1; Sarah Furrows²

${ }_{1}^{1}$ Department of Paediatrics; ${ }^{2}$ Microbiology Department; Kingston Hospital, London, United Kingdom

\section{Background}

Lumbar punctures are carried out in children to exclude meningitis

NICE guidelines recommend consideration of lumbar puncture in:[1]

- Newborns with a CRP rise of $>10 \mathrm{mg} / \mathrm{L}$

- Children $<1$ month with a fever of $>38 \mathrm{C}$

- Febrile children $<3$ months, who are unwell or WCC $<5$ or $>15$

- Other children where meningitis is suspected

Children receive antibiotics pending the results of blood and CSF cultures. Identification of a causative agent can ensure correct treatment, including reduced antibiotic exposure if viral aetiology, and ensure appropriate follow-up testing is arranged. Viral meningitis can be diagnosed with viral PCR, and is traditionally associated with a moderately increased white cell count in the CSF (pleocytosis). However, as is becoming recognised in literature,[2] we are seeing positive PCR results without this pleocytosis.

\section{Methods}

All cerebrospinal fluid (CSF) results at our hospital, which has both neonatal and paediatric wards, were retrospectively analysed fo the significance of their viral PCR testing. CSF WCC, glucose, protein, and viral PCR and bacterial culture results were recorded, along with blood markers and follow-up arrangements.

\section{Results}

There were 996 CSF samples from 939 patients in

the just over 3 years between 13/12/2014-31/1/2018. 511 were from the neonatal ward and 485 were from paediatrics wards. 33 samples (3.3\%) had a positive CSF bacterial growth, growing 44 organisms, most being contaminants. No sample was both vira PCR positive and culture positive.

Viral PCR using a Roche platform was carried out on 519 samples, and was positive in 82 samples (15\% of those tested):

\begin{tabular}{|l|c|}
\hline \multicolumn{1}{|c|}{ Virus } & \# positive \\
\hline Enterovirus & 57 \\
\hline Parechovirus & 21 \\
\hline Herpes Simplex Virus 1 & 2 \\
\hline Herpes Simplex Virus 2 & 1 \\
\hline
\end{tabular}

The majority (55\%) of these PCR-positive samples were from CSF samples without any pleocytosis $\left(\mathrm{CSF} W C C<3 / \mathrm{mm}^{3}\right.$ ), as shown in Figure 1.

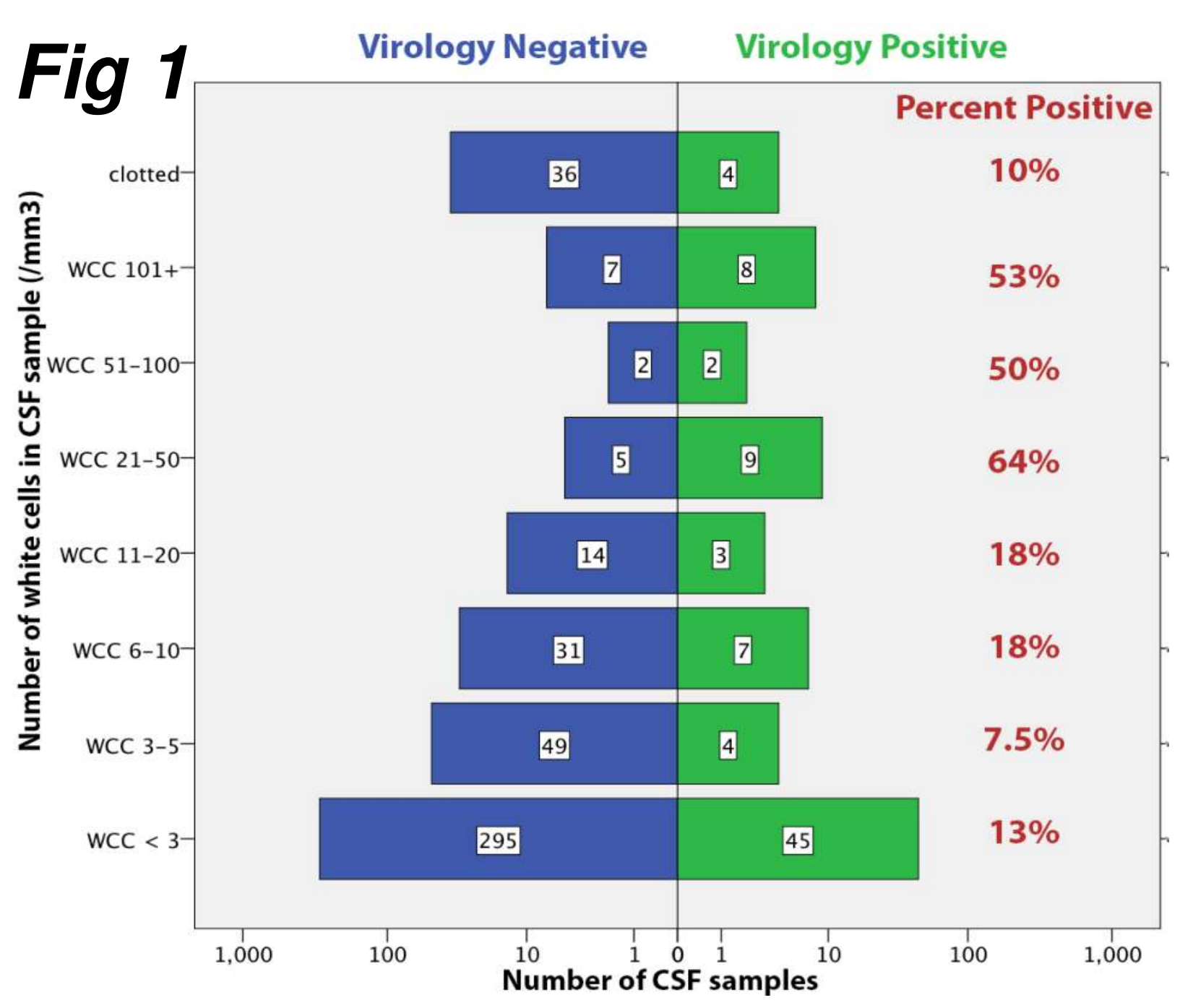

Despite testing viral PCR on 213 samples from babies $<5$ days old, no sample was positive, as shown in Figure 2. If these babies are excluded, the viral PCR positivity rate is $27.2 \%$

With the PCR-positive children, 39/82 (48\%) received audiology testing follow-up for their meningitis. One child (enterovirus at 2 months old, CSF WCC $32 / \mathrm{mm}^{3}$ ) showed an audiological deficit, although it is unclear whether this was due to the meningitis (the patient had failed a prior routine neonatal audiology check).

\section{Discussion}

The traditional view of viral invasion of the CSF as producing a moderately raised CSF WCC is being challenged. Viral RNA/DNA was detected in around 1 in 7 CSF samples without any detectable pleocytosis. The very significant exception to this is in neonates $<5$ days old, where no sample out of $>200$ was positive. Given all of these viruses have at least a $72 \mathrm{~h}$ incubation period, these results fit with viruses expected to be horizontally (rather than vertically) transmitted, and also suggest the positive results without pleocytosis are unlikely to be false positives.

Economically, the viral PCR tests cost GBP £33.39 per test. Each

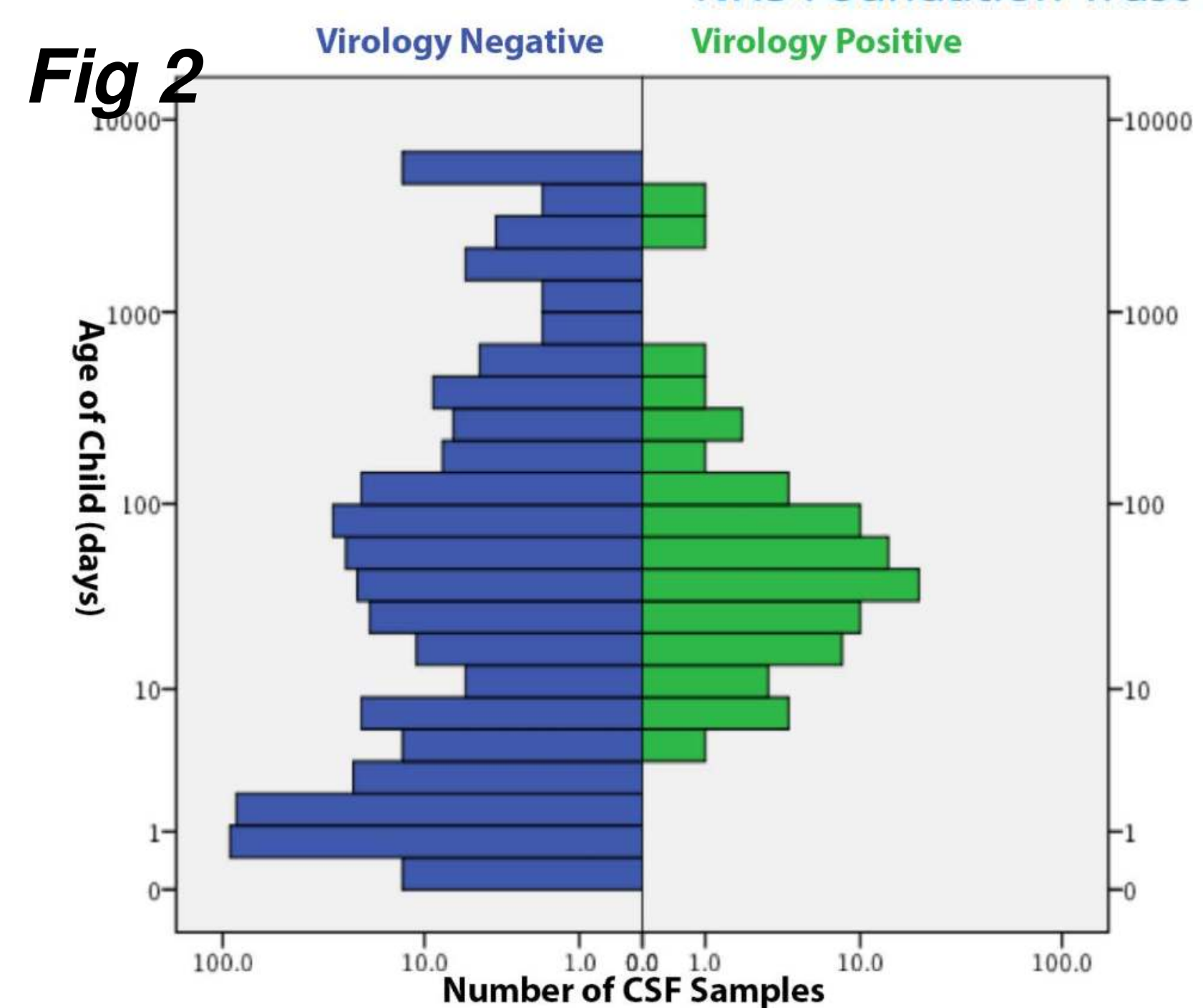

CSF PCR positive result increases coding to a minimum of PA16A (Major Infection with CC, minimum tariff of £2094) per episode. A viral positive PCR gave an average uplift of $£ 1473$ per episode (above the coding without positive PCR). Adding the viral PCR test would pay for itself 6.6 times over, assuming a $15 \%$ positivity rate, or 12 times over if babies $<5$ days are excluded. It also gives a definitive diagnosis to parents and clinicians.

There remains the question of how to follow up children who have detectable viral R/DNA in their CSF without any evidence of inflammation: this could potentially represent a benign viral presence without meningeal irritation. It is unclear whether patients require audiology or other follow-up. Given improved diagnostics, the number of recognised cases are likely to increase. It is an important area for future research to ensure we adequately capture neurological sequelae of these cases.

\section{Conclusions}

Viral R/DNA is frequently found in the absence of pleocytosis Definitive diagnosis can help de-escalate other treatments, and currently has economic benefits.

- It is unclear what follow-up (if any) these children require

- Unless vertical transmission is suspected, there seems to be little case for viral PCR testing neonates under 5 days old 